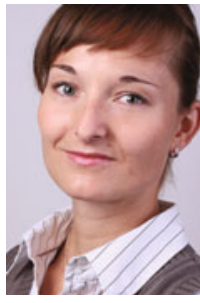

Eva Biegel

2001-2007 Biologiestudium an der Universität Frankfurt a. M. 2003-2004 University of Massachusetts in Dartmouth, MA, USA. 2007-2011 Promotion bei Prof. Dr. V. Müller, Universität Frankfurt a. M. Seit 2011 Research Scientist bei BASF SE, Ludwigshafen.

DOI: $10.1007 / \mathrm{s} 12268-012-0209-5$

(C) Springer-Verlag 2012

Anaerobe, acetogene Bakterien sind in der Natur weit verbreitet und stellen ein essenzielles Glied der anaeroben Nahrungskette dar. Das von ihnen produzierte Acetat setzen methanogene Archaeen $\mathrm{zu} \mathrm{CH}_{4}$ und $\mathrm{CO}_{2} \mathrm{um}$, die in die Atmosphäre entweichen und damit den Kohlenstoffkreislauf schließen. Acetogene Bakterien sind stoffwechselphysiologisch divers und können chemoorganoheterotroph oder chemolithoautotroph durch $\mathrm{CO}_{2}$-Reduktion zu Acetat wachsen. Die $\mathrm{CO}_{2}$-Reduktion zu Acetat geschieht über den Wood-Ljungdahl-Weg (Abb. 1). Seit über 20 Jahren ist bekannt, dass das acetogene Bakterium Acetobacterium woodii diesen Weg mit dem Aufbau eines $\mathrm{Na}^{+}$-Potenzials über der Zytoplasmamembran koppelt. Da dieser Organismus keine Cytochrome oder Chinone enthält, wurde vermutet, dass eines der Enzyme des Kohlenstoffflusses membrangebunden ist. Experimentell konnte dies lange nicht bestätigt werden. Doch vor wenigen Jahren erfolgte die Wende mit der Entdeckung eines membrangebundenen, in den Elektronenfluss involvierten Enzyms, der Ferredoxin:NAD ${ }^{+}-\mathrm{Oxi}^{-}$ doreduktase-Aktivität (Fno).

Ich invertierte Vesikel von $A$. woodii, um herauszufinden, ob der Elektronentransfer von reduziertem Ferredoxin auf $\mathrm{NAD}^{+}$mit einem $\mathrm{Na}^{+}$-Transport über die Membran

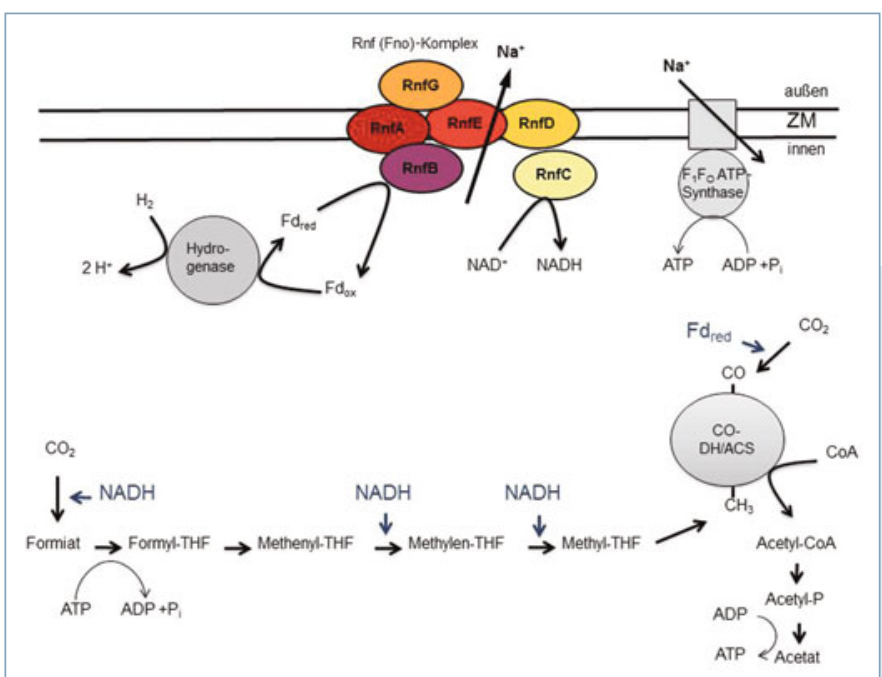

VAAM-Promotionspreis 2012

\section{Energiekonservierung in Acetobacterium}

EVA BIEGEL

BASF, LUDWIGSHAFEN

gekoppelt ist. Die Oxidation von reduziertem Ferredoxin mit gleichzeitiger Reduktion von $\mathrm{NAD}^{+}$führte in der Tat zur Akkumulation von ${ }^{22} \mathrm{Na}^{+}$im Lumen der invertierten Vesikel. Der $\mathrm{Na}^{+}$-Transport ging mit dem Aufbau eines elektrischen Feldes einher, und die Akkumulation von $\mathrm{Na}^{+}$wurde durch $\mathrm{Na}^{+}-$Ionophore, aber nicht durch Protonophore gehemmt, d. h. ein primärer $\mathrm{Na}^{+}$-Gradient durch Fno erzeugt. Diese Untersuchung enthüllt nicht nur die erste an den Stoffwechsel gekoppelte Ionenpumpe in acetogenen Bakterien, sondern zeigt auch zum ersten Mal, dass der exergone Elektronenfluss von Ferredoxin (-500 Millivolt) auf $\mathrm{NAD}^{+}(-320$ Millivolt) mit dem Aufbau eines elektrochemischen Ionengradienten über der Membran einhergeht [1]. Die Natur der Ferredoxin:NAD ${ }^{+}$-Oxidoreduktase konnten wir aufklären. Nach partieller Reinigung des Enzyms und der Identifizierung der Untereinheiten stellte sich heraus, dass diese den Untereinheiten des schon zuvor beschriebenen Rnf-Komplexes aus Rhodobacter capsulatus ähneln. Dieser Komplex ist in die Stickstofffixierung involviert (Rnf: Rhodobacter nitrogen fixation). Die mit molekularbiologischen Methoden detektierten Gene rnfCDGEAB in $A$. woodii codieren für einen membranintegralen Komplex aus sechs nicht-identischen Untereinheiten mit EisenSchwefel-Zentren und Flavinen als Elektronenüberträger. Bioinformatische Methoden wiesen das Vorkommen der $r n f$-Gene in über 200 Genomen bakterieller und archaeeller Spezies nach [2]. Die weite vieler Mikroorganismen.
Verbreitung dieser Gene zeigt, dass der RnfKomplex nicht nur als Ferredoxin-getriebene Ionenpumpe $\left(\mathrm{Na}^{+} / \mathrm{H}^{+}\right)$in anaeroben Mikroorganismen dient, sondern auch die Energie für den rückläufigen, endergonen Elektronentransport von $\mathrm{NAD}^{+}$auf Ferredoxin bereitstellt, wie er z. B. für die Funktion der Nitrogenase wichtig ist.

Diese Befunde bilden den ersten Schritt zur Lösung des Rätsels, wie die $\mathrm{CO}_{2}$-Reduktion zu Acetat mit der Energiekonservierung in acetogenen Bakterien einhergeht. Die Identifizierung einer membrangebundenen $\mathrm{Na}^{+}-$ translozierenden Ferredoxin:NAD ${ }^{+}$-Oxidoreduktase strahlt weit über das Untersuchungsobjekt hinaus. Sie spielt wahrscheinlich eine Rolle in der Energiekonservierung

\section{Literatur}

[1] Biegel E, Müller V (2010) Bacterial $\mathrm{Na}^{+}$-translocating ferredoxin:NAD ${ }^{+}$oxidoreductase. Proc Natl Acad Sci USA 107:18138-18142

[2] Biegel E, Schmidt S, Gonzales J et al. (2011) Biochemistry, evolution and physiological function of the Rnf complex, a novel ion-motive electron transport complex widely distributed in prokaryotes. Cell Mol Life Sci 68:613-634

\section{Korrespondenzadresse: \\ Dr. Eva Biegel \\ BASF SE \\ GVF/H - A030 \\ D-67056 Ludwigshafen \\ Tel.: 0621-60-40847 \\ Fax: 0621-60-6640847 \\ eva.biegel@basf.com}

Sponsoren der vier VAAM-Promotionspreise 2012 waren die Firmen: BASF, Sanofi-Avensis, Bayer Healthcare, New England Biolabs, Evonik Degussa.
Abb. 1: Schema der Energiekonservierung im Zuge des Wood-Ljungdahl-Weges in Acetobacterium woodii. Eine lösliche Hydrogenase reduziert zunächst Ferredoxin (Fd). Die Elektronen werden dann vom reduzierten Ferredoxin über den Rnf-Komplex in der Zellmembran (ZM) auf NAD ${ }^{+}$ übertragen, dabei werden Natrium-Ionen nach außen gepumpt. Durch den elektrochemischen $\mathrm{Na}^{+}$-Gradienten wird über eine $\mathrm{Na}^{+}$-abhängige $\mathrm{F}_{1} \mathrm{~F}_{0^{-}}$ ATP-Synthase ATP gebildet. NADH ist der Elektronendonor für die $\mathrm{CO}_{2}$ Reduktion. Die Natur der Hydrogenase und die Frage, ob weitere Reaktionen des Weges mit der Reduktion von Ferredoxin einhergehen, ist Gegenstand derzeitiger Untersuchungen. THF: Tetrathydrofolat; CO-DH/ACS: Co-Dehydrogenase/Acetyl-CoA-Synthase. 